\title{
Panniculitides in Rheumatoid Syndromes: The Role of Histopathology
}

\author{
Carlo Tomasini \\ Department of Clinical and Surgical, Diagnostic and Pediatric Sciences, Dermatology Clinic, \\ University of Pavia and IRCCS Policlinico San Matteo, Pavia, Italy
}

Keywords

Panniculitis · Rheumatoid syndromes · Histopathology

The diagnosis of panniculitis is considered the "ugly duckling" for clinicians and pathologists. Clinically, the patients usually present with erythematous subcutaneous nodules with a quite monotonous appearance and often without additional symptoms; histopathologically, the subcutaneous fat responds to a variety of insults in a limited number of forms, and pathologic differences between some panniculitides are sometimes subtle or overlapping. Although the biopsy plays a critical role in the diagnostic process of a panniculitis, pitfalls that inevitably will limit the diagnostic value of this procedure may be numerous, usually arising at the interface between clinician and dermatopathologist [1,2]. Sampling error is perhaps the most common one. Indeed, inadequate biopsy specimens are often obtained, commonly being too superficial in nature or too narrow in breadth to render a diagnosis with certainty. Poor diagnostic clinical skill combined with a faulty biopsy may direct to a completely erroneous diagnosis. The basic prerequisites of panniculitis are summarized in Table 1.

The working approach to histopathologic diagnosis of panniculitis relies on a "step-bystep" method (Table 2). Once the inflammatory reaction is recognized to be panniculitis on scanning microscopic examination, the pathologist attempts to further classify the panniculitis according to the preferential localization of the cellular infiltrate, i.e., if it affects primarily the fat lobules or the septa between the lobules. The next step refers to examination of blood vessels to determine as to whether coexisting vasculitis is present or not, and if vasculitis is detected, the size and type of the vessels involved must be determined. The following step is 
Table 1. Prerequisites to get the most out of useful information from a biopsy of panniculitis

A large and deep incisional biopsy is preferred (for some conditions, e.g., deep morphea and eosinophilic fasciitis, tissue should also include the fascia).

Punch biopsy is of second choice. In this case, use punches of $6 \mathrm{~mm}$ or more. Avoid small punches as subcutaneous fat is generally not included in the tissue removed or, even though a small bit of fat is included, it often retracts away from the dermis during extraction. Be aware that a sufficient portion of subcutaneous fat is included in the biopsy.

Select early evolving inflammatory lesions by a careful clinicopathologic correlation. Avoid late-stage lesions as they show nonspecific findings.

Prefer lesions above the knee, as effects of venous stasis may distort the histopathologic appearance of primary pathology.

If an infectious process is suspected, a biopsy for fresh tissue culture and/or PCR study should be considered.

If a lymphoproliferative disease is suspected, additional biopsies for extended immunophenotyping on frozen section and PCR for lymphocyte clonality studies are recommended.

If an autoimmune pathogenesis is suspected (e.g., lupus erythematosus, rheumatoid arthritis), a biopsy for direct immunofluorescence is recommended.

Discuss with the dermatopathologist prior to performing the biopsy if there is any uncertainty about the ideal biopsy, type, or fixation/storage methods and ancillary techniques required.

Table 2. Algorithmic approach to histopathologic diagnosis of panniculitides

- Identify the location of the most intense inflammatory infiltrate (septa or lobules)

- Determine whether it is vasculitis or not

- Determine the composition/distribution of the inflammatory infiltrate

- If lymphocytes predominate, the degree of cytologic atypia should be assessed

- Look for clues and/or specific changes

- Integrate everything with the clinical and laboratory findings

the evaluation of the type and quality of the inflammatory infiltrate; if lymphocytes are predominant, the degree of cytologic atypia should be assessed, as subcutaneous panniculitis-like T-cell lymphoma or another type of lymphoma may mimic "lymphocytic" panniculitis. The application of this algorithmic method may simplify the histopathologic approach to panniculitis; nevertheless, it could be a limit as it does not consider different stages in the evolution and/or exceptions to the rule (e.g., cases of erythema nodosum with predominant lobular involvement).

Rheumatoid-associated panniculitides are varied and encompass a number of entities; some of them define the dominant clinical features (e.g., the rheumatoid nodule or subcutaneous cords), while other allude to histopathology (e.g., rheumatoid neutrophilic panniculitis). According to the dominant histopathologic reaction pattern in the subcutaneous fat, three main categories may be defined, namely palisading and/or interstitial granulomatous panniculitis (Fig. 1), neutrophilic panniculitis (Fig. 2), and panniculitis with vasculitis (neutrophilic, lymphocytic, granulomatous) (Fig. 3) [3]. The different morphology reflects the dominant immunologic mechanisms that are operative in the pathogenesis of a particular rheumatoid syndrome, including immune complex activation of the complement cascade to 
Fig. 1. Palisading and interstitial granulomatous panniculitis. a Railway track-like subcutaneous cords on the lateral chest wall in a patient with rheumatoid arthritis. b Interstitial and palisaded granulomatous infiltrate within the deep reticular dermis and subcutaneous fat. c The infiltrate consists of histiocytes and polymorphonuclear cells. d Tiny foci of severe basophilic collagen degeneration surrounded by histiocytes and nuclear dust (ChurgStrauss granuloma in miniature).

Fig. 2. Neutrophilic panniculitis. a Erythematous nodule on the thigh in a patient with rheumatoid arthritis. b Lobular and septal neutrophilic panniculitis. c The lobule is predominantly involved with fat necrosis. d Neutrophils and nuclear dust.
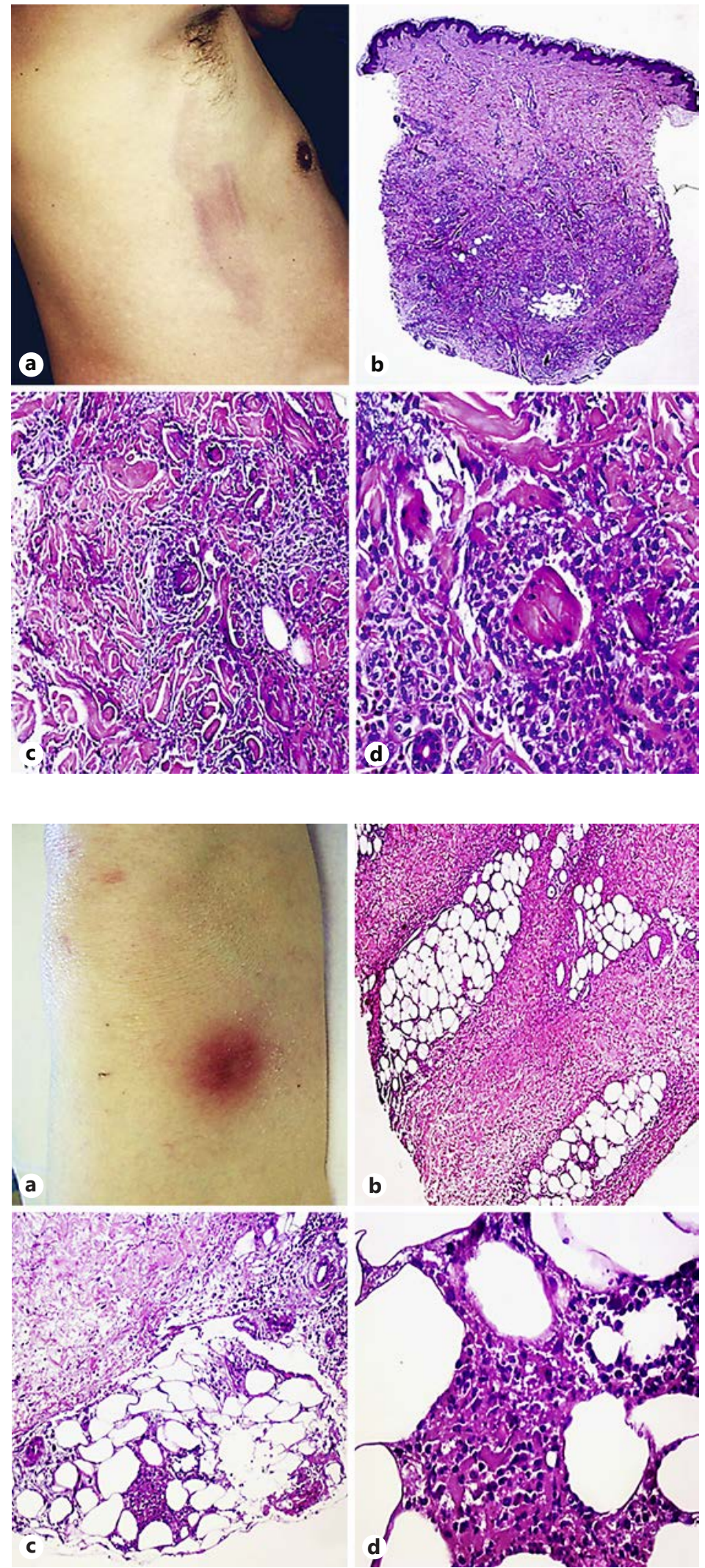
Fig. 3. Panniculitis with vasculitis. a Subcutaneous nodules on the upper arm in a patient with rheumatoid arthritis. b Palpable purpura with nodules on the lower extremity. c Neutrophilic panniculitis with vasculitis (nodular vasculitis).

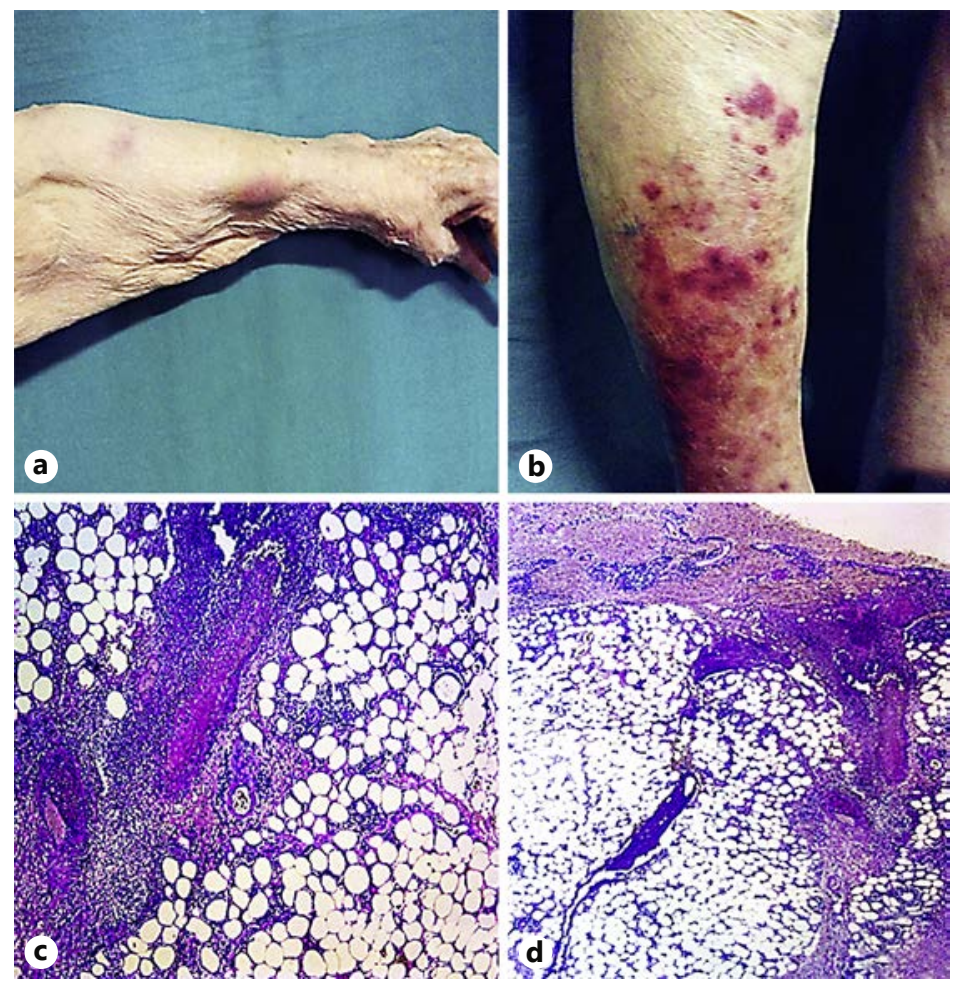

generate neutrophilic chemotactic factors and immune-complexes deposition in the vessel walls with variable ischemic tissue damage ("necrobiosis") and granuloma formation. Any one pattern may predominate, but overlap among them may be expected. Clinicopathologic correlation is required for definite diagnosis.

\section{Statement of Ethics}

The author has no conflicts of interest to disclose.

\section{Disclosure Statement}

The author declares no conflicts of interest.

\section{References}

1 Requena L: Normal subcutaneous fat, necrosis of adipocytes and classification of the panniculitides. Semin Cutan Med Surg 2007;26:66-70.

2 Tomasini C, Lentini F, Borroni G: Skin biopsy in diagnosis of panniculitides. G It Dermatol Venereol 2013;148: 335-349.

3 Magro CM, Crowson AN: The spectrum of cutaneous lesions in rheumatoid arthritis: a clinical and pathological study of 43 patients. J Cutan Pathol 2003;30:1-10. 